\title{
COMENTARIO JURÍDICO A LA RECOMENDACIÓN GENERAL 29 "SOBRE EL EXPEDIENTE CLÍNICO COMO PARTE DEL DERECHO A LA INFORMACIÓN EN MATERIA DE SALUD” EMITIDA POR LA CNDH
}

\author{
Alejandro DÍAZ PÉREZ* \\ Rita Astrid MUCIÑO CORRO**
}

\section{INTRODUCCIÓN}

El 31 enero de 2017, la Comisión Nacional de los Derechos Humanos (en adelante la Comisión o CNDH) emitió la Recomendación General 29 "Sobre el expediente clínico como parte del derecho a la información en materia de salud". Lo hizo de acuerdo con la atribución contenida en la fracción VIII del artículo 6o. de su Ley, que la faculta para proponer que se promuevan los cambios y modificaciones de leyes y reglamentos, así como de prácticas administrativas, que procuren y garanticen una mejor y más amplia protección de los derechos humanos a las diversas autoridades del país en el exclusivo ámbito de su competencia.

Esta decisión tiene una profunda importancia, por varias razones. Por una parte, la temática específica del documento es única en su tipo en

* Licenciado en derecho y ciencias sociales, Universidad Michoacana de San Nicolás de Hidalgo. Especialista en derechos humanos y máster en derecho constitucional por la Universidad de Castilla-La Mancha, España. Máster en derechos humanos por la Universidad Autónoma de Madrid, España. Exvisitante profesional en la Corte Interamericana de Derechos Humanos, en San José, Costa Rica. Actualmente, abogado del Grupo de Recomendaciones de la Cuarta Visitaduría General de la Comisión Nacional de los Derechos Humanos.

** Maestra en derechos humanos y democracia por la Facultad Latinoamericana de Ciencias Sociales, con especialidad en derechos humanos por la Universidad Castilla-La Mancha. Fue Visitante profesional en la Corte Interamericana de Derechos Humanos

Fecha de recepción: 24 de enero de 2019.

Fecha de dictamen: 28 de marzo de 2019. 
México, en tanto contiene elementos de análisis sobre la forma en cómo el expediente clínico es una vía directa para garantizar el derecho a la información en el ámbito de la salud.

Asimismo, desde otra perspectiva, la motivación de la Comisión para emitir la Recomendación General guarda relación con la cuantiosa evidencia empírica, basada en 96 casos ocurridos entre 2010 y 2016, en los que diversas autoridades públicas incumplieron con la debida integración del expediente clínico (CNDH, 2017a: 5), al tiempo que da cuenta de las dificultades existentes en el sistema de sanidad del país para el tratamiento de los datos personales en el ámbito médico.

A partir del estudio de dichos casos, el organismo de derechos humanos identificó el posible origen de las problemáticas existentes y formuló una serie de recomendaciones, que pudieran servir para solucionar aquellos hechos violatorios que dan lugar al menoscabo del derecho a la información en materia de salud, entre otros.

El presente comentario jurídico pretende realizar una revisión crítica de la Recomendación General 29. Se partirá de los enfoques de derechos humanos y de género para sostener que no obstante su indudable importancia, ésta cuenta con algunos puntos "ciegos"; que tendrán que ser reorientados, profundizados y, en su caso, reformulados.

De esta manera, los argumentos del texto se construyen sobre criterios de revisión relacionados con aspectos del derecho de acceso a la información aplicables a la integración de expedientes médicos, la protección de datos personales en posesión de médicos e instituciones de salud pública y privada, la titularidad y propiedad del mismo, el plazo para la conservación del expediente clínico, la vulnerabilidad de la información y medidas de seguridad, así como la importancia de abordar la temática desde la perspectiva de género.

\section{Recomendación General 29 "Sobre el expediente CLÍNICO COMO PARTE DEL DERECHO A LA INFORMACIÓN EN MATERIA DE SALUD”}

La justificación de la emisión de la Recomendación General 29 encuentra su base en al menos dos cuestiones sustanciales. La primera, relacionada con la documentación de omisiones sistemáticas (CNDH, 2017a: 5) por parte del personal de salud de diversas instituciones en la elaboración de los expedientes clínicos de las y los pacientes; y segunda, en el carácter incremental (CNDH, 2017a: 6), de este tipo de vulneraciones a lo largo de los 
últimos años. La suma de estos factores permite inferir la razón por la que ésta se dirigió a prácticamente todas las instituciones de salud del Estado mexicano, en los diversos órdenes de gobierno. ${ }^{1}$

El análisis parte de los antecedentes que dieron forma a los desarrollos normativos del expediente clínico. Al respecto, éste se implementó desde 1986, pasando por una reforma en 1993, para finalmente expedirse en 2012 la Norma Oficial Mexicana NOM-004-SSA3-2012, "Del Expediente Clínico" (en lo sucesivo NOM-004).

Asimismo, países como Estados Unidos, España, Francia, Bélgica, Argentina, Costa Rica, Colombia y Uruguay (CNDH, 2017a: 13-19) tienen importantes avances jurídicos, lo que da cuenta de la prevalencia de un cierto consenso a nivel comparado de la inclusión del expediente clínico o historia clínica como un elemento del derecho a la información.

Ahora bien, respecto al análisis central de la integración del expediente clínico como parte del derecho a la información en materia de salud, debe partirse de la inevitable interrelación del derecho a la protección de la salud (en sentido amplio) previsto en el artículo 4o. de la Constitución Política de los Estados Unidos Mexicanos, con el derecho a la información contemplado en el artículo 6o. del mismo ordenamiento.

Sumando a lo anterior, el Comité de Derechos Económicos, Sociales y Culturales de la ONU (Comité DESC) ha interpretado que en materia de salud el derecho a la información comprende "el derecho de solicitar, recibir y difundir información e ideas acerca de las cuestiones relacionadas con la salud. Con todo, el acceso a la información no debe menoscabar el derecho de que los datos personales relativos a la salud sean tratados con confidencialidad" (Comité DESC, 2000: 12).

De forma más específica, del expediente clínico y su relación con el derecho a la información han sido abordados por la Corte Interamericana de Derechos Humanos (Corte IDH), al referir que "un expediente médico, adecuadamente integrado, es instrumento guía para el tratamiento médico, y fuente razonable de conocimiento acerca de la situación del enfermo, las medidas adoptadas para controlarla y, en su caso, las consecuentes responsabilidades" (caso Albán Cornejo y otros vs. Ecuador: 68).

A nivel interno, a decir de la Comisión, la Ley General de Salud contempla al expediente clínico en una doble dimensión: 1) como una obli-

1 Secretarios de Salud, de la Defensa Nacional y de Marina; gobernadores de las entidades federativas; jefe de gobierno de la Ciudad de México, y directores generales del Instituto Mexicano del Seguro Social, del Instituto de Seguridad y Servicios Sociales de los Trabajadores del Estado y de Petróleos Mexicanos. 
gación vinculada con el principio de calidad ${ }^{2}$ en el servicio, y 2) como un derecho contenido en el artículo 77 bis $37^{3}$ (CNDH, 2017a: 30).

Bajo esta perspectiva, el derecho a la información en materia de salud, relativo al expediente clínico de las personas, es "aquella libertad atribuible a los usuarios de servicios médicos para solicitar, recibir y conocer todas las constancias y elementos que integran dicho expediente clínico"(CNDH, 2017a: 32), y estaría conformada por al menos tres aspectos fundamentales: 1) el acceso para recibir todo tipo de información relacionada con la atención de la salud; 2) la protección de los datos personales, y 3 ) la información en disposición bajo los principios de accesibilidad, confiabilidad, verificabilidad, veracidad y oportunidad (CNDH, 2017a: 34).

No obstante este cúmulo de previsiones normativas, cabe preguntarse en dónde se concentran las problemáticas relevantes sobre la cuestión. En un primer momento, de acuerdo con la propia recomendación general de la CNDH, las omisiones más recurrentes sobre la integración del expediente clínico — que pudieran llamarse formales - se refieren al incumplimiento de los requisitos contenidos en la NOM-0042, relacionados con el asentamiento de datos, como hora, fecha, y nombre de quienes realizan las notas médicas, datos de exploración y tratamientos médicos, notas de evolución, hojas de enfermería, entre otros (CNDH, 2017a: 39).

En otro sentido, concurren problemas que son de índole estructural; esto es, la existencia de un vínculo entre las condiciones laborales del personal médico que dificulta la manera en que se asientan con debida diligencia los datos del expediente clínico, y la existencia de un problema en la lógica de funcionamiento de las instituciones de salud (CNDH, 2017a: 42).

A su vez, la Comisión agrega que en relación con la práctica médica, "la sobrecarga de trabajo derivada del alto número de pacientes que deben atender en poco tiempo, haciendo materialmente imposible su debida integración"; es decir, alude a un criterio pragmático, al tiempo que enfatiza la existencia de "extenuantes jornadas laborales que surgen como consecuencia del entendimiento de la cultura del trabajo en las instituciones de salud y la visión social generalizada del trabajo y práctica médica” (CNDH, 2017: 43). En otra línea, subraya la prevalencia de "deficiencias de infraes-

2 Por calidad, el Comité DESC ha descrito que los servicios de salud deben ser culturalmente adecuados y aceptables tanto científica como médicamente. Ello requiere, entre otras cosas, personal médico capacitado, medicamentos y equipo hospitalario científicamente aprobados y en buen estado, agua limpia potable y condiciones sanitarias adecuadas.

3 Artículo 77 bis 37: "Los beneficiarios del Sistema de Protección Social en Salud tendrán además los derechos establecidos en el artículo anterior, los siguientes... VII. Contar con su expediente clínico...”. 
tructura, recursos tecnológicos y la falta de implementaciónn de sistemas automatizados o electrónicos" (CNDH, 2017: 44).

Como formas de solución a estos obstáculos, el organismo nacional plantea distintos niveles de aproximación. Al respecto, sugiere cinco medidas, a saber: 1) la adopción de medidas administrativas, financieras, jurídicas o de cualquier otra índole, para que se procure el debido cumplimento en la integración de los expedientes clínicos; 2) la realización de estudios que permitan advertir cuál es la relación de causalidad existente entre las condiciones laborales del personal médico y la indebida integración de los expediente clínicos; 3) se proporcionen los recursos tecnológicos necesarios que faciliten al personal de salud la debida integración del expediente clínico; 4) desde el ámbito institucional o intersectorial, se articulen asimetrías regulatorias que permitan vigilar el cumplimiento de la NOM-004, y 5) la impartición de cursos de capacitación y formación en materia de derechos humanos, así como del conocimiento, manejo y observancia de las normas oficiales mexicanas del expediente clínico (CNDH, 2017a: 20 y 21).

Habida cuenta de estas propuestas de posible solución en el tratamiento de los datos e información contenida en los expedientes clínicos, se advierte que existen aspectos que la Recomendaciónn General 29 debió cuestionar, y que deben incorporarse en futuros esfuerzos, al tiempo que considerarse por las diversas instituciones de salud pública del país, como a continuación se analiza.

\section{Los VACíos QUE DEJA LA RECOMENDACióN General 29}

En un primer momento, puede identificarse que la Recomendación General no fue construida a la par de organizaciones de la sociedad civil, instituciones académicas en el ámbito médico y/o con órganos autónomos pares a la CNDH, como el Instituto Nacional de Transparencia, Acceso a la Información y Protección de Datos Personales (INAI). Lo anterior hubiera permitido concentrar todas las voces que pudieran aportar al debate público sobre la temática en cuestión. Sobre ello, el documento no hace ninguna referencia a este proceso de socialización del contenido de la Recomendación. ${ }^{4}$

4 Sobre la relevancia de enriquecer estos procesos institucionales con los aportes de la sociedad civil y otros organismos no jurisdiccionales, los Principios de París disponen que en el marco de sus actividades, las instituciones nacionales deben: "6. mantener la coordinación con los demás órganos de carácter jurisdiccional o de otra índole encargados 
Asimismo, no se advierte que en la resolución se haya tenido en cuenta la forma en que esto sucede en las entidades federativas, cuestión que hubiera sumado al entendimiento de dicho fenómeno, al brindar un panorama más amplio acerca de las circunstancias que imperan tanto en los centros de salud estatales como en los municipales, al igual que en los ubicados en zonas rurales - siendo éstos donde se concentra el mayor número de población susceptible de verse afectada por este problema-.

Por otra parte, avanzando en los aspectos propios de la resolución, no contempla a la Ley General de Transparencia y Acceso a la Información Pública, y/o a la Ley Federal de Transparencia y Acceso a la Información Pública como un marco de referencia o fuente de obligación para las diversas instituciones del Estado mexicano en relación con el expediente clínico.

Asimismo, un aspecto relevante que fue omitido tiene que ver con el desarrollo de las obligaciones existentes sobre el derecho a la información en materia de salud, cuando se trata de protección frente a terceros o particulares. En este punto, la Ley Federal de Protección de Datos Personales en Posesión de los Particulares 5 y su Reglamento debió ser abordada por la Comisión como un marco esencial.

También debe recordarse que de acuerdo con las normas de derechos humanos, las autoridades de un Estado pueden ser responsables por cualquier violación a los derechos humanos cometida por particulares que ocurra dentro de su jurisdicción si devienen tres condiciones: i) al momento de los hechos existe una situación de riesgo real e inmediato; ii) las autoridades conocían o debían tener conocimiento de dicha situación, y iii) no adoptar las medidas razonables y necesarias para prevenir o evitar ese riesgo (caso Rodríguez Vera y otros vs. Colombia: 523).

Esta cuestión cobra suma relevancia en tanto que aproximadamente el 24\% de las personas en México son usuarios del sector privado de salud (Inmujeres, 2010), cuyos datos personales contenidos en los expedientes

de la promoción y protección de los derechos humanos...; 7. establecer relaciones con organizaciones no gubernamentales que se ocupen de la promoción y protección de los derechos humanos...". Principios de París, adoptados por la Asamblea General de las Naciones Unidas en 1993. Por su parte, el artículo 51, inciso B), fracciones I a III del Reglamento de la CNDH, establece que se deberán impulsar los procesos de colaboración y consolidar la relación con las organizaciones de la sociedad civil para la implementación de una agenda de derechos humanos. Reglamento Interno de la Comisión Nacional de los Derechos Humanos, última reforma incorporada: 22 de diciembre de 2017.

5 Al respecto, la fracción VI del artículo 3 de dicha Ley hace referencia al estado de salud presente y futuro de una persona como un dato personal sensible. 
clínicos son tratados por médicos y hospitales privados, y que exigen una actuación del Estado frente a esta circunstancia.

Otro elemento que merece una reflexión particular es el relacionado con la titularidad o propiedad del expediente clínico. De acuerdo con el numeral 5.4 de la NOM-004, los expedientes clínicos "son propiedad de la institución o del prestador de servicios médicos que los genera”. Al respecto, esta posición es problemática, y debe cambiarse para transitar hacia una que contenga un enfoque de derechos humanos más apropiada, en tanto la propiedad (y titularidad) del expediente debe ser de la persona que recibe el servicio de salud, no sólo la información en sí misma, sino del objeto como tal. ${ }^{6}$ Por esta razón, la Comisión en futuras decisiones debería profundizar sobre este aspecto, y no abstenerse, como en la Recomendación General 29.

Sobre el particular, resulta pertinente hacer mención de lo sostenido por la Segunda Sala de la Suprema Corte de Justicia de la Nación (SCJN) en el amparo en revisión 632/2014, en el que entre otros puntos refirió que los numerales 5.4 a 5.7 de la NOM-004 "deben interpretarse en un sentido amplio", de forma que la persona usuaria tenga la posibilidad de allegarse, cuando así lo solicite, de "cualquier constancia que obre dentro de su expediente, incluso de que éste sea proporcionado en su integridad" (AR 632/2014: 14).

En conexión con lo anterior, la propia NOM-004 prevé que los expedientes clínicos, "por tratarse de documentos elaborados en interés y beneficio del paciente, deberán ser conservados por un periodo mínimo de 5 años, contados a partir de la fecha del último acto médico" (NOM-004: 5.4). Las discusiones sobre el plazo contemplado deberían estar sujetas a un abordaje mayor, y orientadas en incorporar algunas excepciones procedentes para los fines que sean relevantes. ${ }^{7}$

Un aspecto medular adicional lo constituye el hecho de que si bien existe un deber específico de integrar el expediente clínico de cada paciente, esa obligación no implica necesariamente que éste sea electrónico. En este aspecto, la Comisión debió solicitar que se reformara la NOM-004, para que la obligación de empezar a diseñar expedientes electrónicos quedara plasmada. Tal avance facilitaría la conservación de datos personales,

6 El INAI en el criterio 317/17 determinó que la titularidad de los datos personales contenidos en expediente clínico es del paciente y no de hospitales o médicos.

7 Por ejemplo, en casos en los que la información del expediente en cuestión sea relevante para una investigación científica, así como en casos de violaciones de derechos humanos especialmente graves, como serían las prácticas sistemáticas de esterilizaciones forzadas o en abusos sexuales en contextos de revisiones ginecológicas. 
la disminución de la posibilidad pérdidas, robos, extravíos, acumulación de papelería y facilidad de almacenamiento, en contraste con las vías tradicionales de instrumentación del expediente clínico (Moreddu, 2017).

Asimismo, la implementación del expediente clínico electrónico, como instrumento de protección y garantía del derecho a la información, también implicaría una reestructuración profunda de la Norma Oficial Mexicana NOM-024-SSA3-2012, "Sistemas de información de registro electrónico para la salud. Intercambio de información en salud", que establece los objetivos funcionales y funcionalidades que deberán observar los productos de sistemas de expediente clínico electrónico para garantizar la interopera- bilidad, procesamiento, interpretación, confidencialidad, seguridad y uso

- de estándares y catálogos de la información de los registros electrónicos en salud.

Finalmente, la sofisticación de los medios de ingreso de los datos contenidos en un expediente clínico electrónico que se dirijan a disminuir la vulnerabilidad de la información, como la utilización de la firma electrónica avanzada por parte del personal médico autorizado que brinda la atención médica, son un tema central de progresiva inclusión.

\section{Derecho a la información en materia sexual y reproductiva}

La vulneración al derecho a la información en materia sexual y reproductiva es una constante que afecta principalmente a mujeres y niñas. La prevalencia de factores que restringen el goce efectivo del mismo genera consecuencias que les impiden a aquéllas actuar con autonomía, al igual que tomar las decisiones que mejor correspondan a su proyecto de vida.

Por tal motivo, cobra especial relevancia el conocimiento sobre los datos inherentes al estado de salud durante el proceso del embarazo, así como en las etapas de alumbramiento y posteriores, toda vez que se trata de determinaciones sobre salud sexual y reproductiva, así como sobre la vida de las mujeres y el producto de la gestación.

Dicha situación ha dado lugar a numerosas recomendaciones emitidas por la CNDH, e incluso motivó las recomendaciones generales 4/2002 "Derivada de las prácticas administrativas que constituyen violaciones a los derechos humanos de los miembros de las comunidades indígenas respecto de la obtención de consentimiento libre e informado para la adopción de métodos de planificación familiar", y 31/2017 "Sobre la violencia 
obstétrica en el sistema nacional de salud", ${ }^{8}$ en las que constató que el personal de salud, al obtener el consentimiento sobre los métodos de planificación familiar, incurre en una serie de acciones u omisiones, transgresoras de los derechos humanos de las personas usuarias.

De esta manera, es posible afirmar que la CNDH cuenta con la evidencia suficiente para sostener que en la práctica médica es recurrente el incumplimiento de los estándares relacionados con este derecho. No obstante lo anterior, fue omisa en pronunciarse al respecto.

La Recomendación General objeto de estudio representaba una excelente oportunidad para visibilizar las barreras que las mujeres enfrentan al acceder a la información en el contexto de la atención a la salud, particularmente durante la atención del embarazo, del parto y del puerperio, así como para establecer medidas idóneas, considerando que este tipo de actos, más allá de ser resultado de la precaria dinámica organizacional en los hospitales, tienen por origen la discriminación.

Si bien en el mencionado documento se hace referencia a la importancia que una correcta integración revierte para las mujeres víctimas de violaciones a los derechos sexuales y reproductivos, la CNDH sólo realiza una mención superficial, y deja de lado una lectura más profunda que considerara los enfoques de género e intercultural.

No pasa desapercibido el hecho de que la inobservancia de los estándares trae consigo una afectación adicional tratándose de personas provenientes de grupos de atención prioritaria, tales como personas mayores, migrantes, etcétera; sin embargo, de la revisión de los 96 casos analizados por la CNDH en su Recomendación General se identificó que en 61 de ellos las personas afectadas son mujeres, de las cuales

- El 15.8\% es indígena.

- El 15.8\% era menor de edad.

- El $68.2 \%$ estaba embarazada. ${ }^{9}$

En este tenor, la Comisión Interamericana de Derechos Humanos (en adelante la CIDH), en su informe sobre "Acceso a la información en mate-

8 Los casos que se registraron en la Recomendación General 31/2017 datan desde 2015, por lo que el hecho de que se haya emitido con posterioridad a la Recomendación General 29/2017 no exime a la CNDH de haber integrado en esta última un apartado para analizar la vulneración al acceso a la información en material sexual y reproductiva.

9 Para obtener el porcentaje se consideró el número de mujeres que fueron víctimas en los 61 casos, lo cual suma un total de 63. Esa información fue sistematizada de la revisión de las recomendaciones emitidas por la CNDH, que puede consultarse en: http://www. cndh.org.mx/Recomendaciones. 
ria reproductiva desde una perspectiva de derechos humanos", reconoció que las mujeres latinoamericanas enfrentan obstáculos que les impiden ejercer plenamente al multicitado derecho. Asimismo, indicó que los Estados deben asegurar el acceso a la información, así como: a) el consentimiento informado; $b$ ) la protección de la confidencialidad; c) la obligación de entregar información oportuna, completa, accesible, fidedigna y oficiosa; d) el acceso a la historia médica, y e) la obligación de producción de estadísticas confiables (CIDH, 2011: 42).

El primer elemento implica brindar con oportunidad y de manera oficiosa la información necesaria, cerciorarse de que la persona usuaria comprendió cuáles son sus opciones y consecuencias de las mismas, y verificar que no existan vicios en el consentimiento.

De acuerdo con lo establecido por la CNDH en la Recomendación General 4/2002, por lo general la obtención del consentimiento de mujeres indígenas sucede en un escenario en el que no se atiende ninguno de estos aspectos. Sobre el particular, señala la falta de pertinencia cultural de los formatos mediante los cuales se busca recabar la firma, que además de encontrarse redactados sólo en español —idioma que dominan de manera escasa o nula- (CNDH, 2002: 11) en muchas ocasiones se encuentra firmado como persona testigo la o el profesional de salud, circunstancia que pone en duda si en efecto se respetó la autonomía de la persona usuaria.

Con relación a este punto, la Comisión, en la Recomendación General 31/2017, manifestó que de los 28 expedientes en los que se acreditó que hubo violencia obstétrica, en nueve se configuró, al haberse violado el derecho a la información y al libre consentimiento (CNDH, 2017b: 99).

El segundo elemento exige el respeto del secreto profesional, en virtud del cual el personal de salud no puede ser criminalizado por abstenerse de revelar información de la o el paciente.

La confidencialidad asegura que la persona pueda manifestar con la confianza suficiente los datos necesarios para emitir un diagnóstico adecuado. Este componente revierte especial importancia tratándose de las y los adolescentes, a quienes, de acuerdo con el Comité DESC, se les debe respetar su confidencialidad y su vida privada (Comité DESC, 2000: 23).

En cuanto al tercer inciso, la CNDH ha conocido de casos en los que la falta de oportunidad, accesibilidad, veracidad y oficiosidad se han configurado a través de conductas que van desde la negativa de brindar información sobre el estado de salud de la persona internada, hasta la esterilización forzada de la mujer. 
Por lo que se refiere al acceso a la información y a la historia médica, la SCJN ha señalado que las autoridades deben facilitar a la o el paciente, copias completas del expediente clínico, y no limitarse a entregar sólo un resumen de este último (AR 632/2014: 14).

Por último, respecto del acceso a la información en relación con la obligación de producción de estadísticas confiables, es preciso señalar que se trata de un deber que se refuerza tratándose de personas en situación de vulnerabilidad, ya que es una forma de identificar las manifestaciones de la desigualdad, discriminación y violencia de que pueden ser víctimas. Al respecto, la Convención Interamericana para Prevenir, Sancionar y Erradicar la Violencia contra la Mujer (Belem do Pará) conmina al Estado a contar con estadísticas e indicadores para medir el impacto de la violencia, al igual que la efectividad de las medidas adoptadas para su erradicación (artículo 8o.).

En este sentido, se valora positivamente el tratamiento que la CNDH comenzó a darle a la violencia ejercida contra la mujer durante la atención médica recibida en el embarazo, a la que denominó "violencia obstétrica", toda vez que de esta manera es posible dimensionar la frecuencia y detectar la forma en como ocurre; todo lo cual servirá en el diseño de políticas públicas orientadas a mejorar los servicios de salud.

\section{Reflexiones Finales}

El expediente clínico constituye una herramienta fundamental para la persona usuaria, ya que representa un medio para satisfacer diversas necesidades de la o el paciente. Por un lado, es un componente esencial para asegurar el acceso a los servicios de salud de manera oportuna y efectiva, y por el otro, sirve para evidenciar la acción u omisión cometidas por el personal de salud, cuestión que resulta relevante cuando el asunto trasciende al ámbito de lo legal.

En este contexto, la Recomendación General 29 de la CNDH se torna en un importante referente, al basarse para su conformación, en la amplia evidencia empírica disponible, y que se posiciona como único en su tipo, en órganos de derechos humanos en México, y con toda probabilidad en el mundo; también sirve como punto de partida para avanzar en las discusiones contemporáneas sobre el derecho a la información en materia de salud.

No obstante lo anterior, existen varios elementos que dicha recomendación dejó en el olvido, y que deberían retomarse en posteriores decisiones de la Comisión sobre este temática. 
Estos aspectos específicos parten desde la inclusión de marcos normativos, como la Ley General de Transparencia y Acceso a la Información Pública, y/o la Ley Federal de Transparencia y Acceso a la Información Pública; el desarrollo de las obligaciones existentes sobre el derecho a la información en materia de salud, cuando se trata de protección frente a terceros o particulares (deber de proteger), la modificación del enfoque de titularidad y propiedad del expediente clínico, los periodos temporales de su conservación, hasta la obligación de implementar expedientes electrónicos, a través de una profunda reforma de las normas oficiales, y la generación de mecanismos que se dirijan a disminuir la vulnerabilidad de la información.

Con la introducción de estos múltiples elementos al debate podrían visibilizarse de mejor forma aquellas cuestiones que son especialmente problemáticas para el acceso y tratamiento de los datos personales contenidos en los expedientes clínicos, y que en consecuencia pueda ser mejor garantizado el derecho a la información en materia de salud.

Finalmente, la CNDH, como organismo protector de los derechos humanos, no puede dejar al margen a las personas pertenecientes a los grupos de atención prioritaria. En indispensable que al abordar la situación jurídica y contextual de un derecho humano en aras de emitir una recomendación general profundice en los agravios que el incumplimiento de las obligaciones estatales trae para las y los integrantes de estos grupos, sin soslayar la manera en que la interacción de las diferentes condiciones dificulta aún más el ejercicio pleno del derecho a la información.

\section{Fuentes COnsultadas}

Comisión Interamericana de Derechos Humanos (CIDH), 2011, "Acceso a la información en materia reproductiva desde una perspectiva de derechos humanos".

Comisión Nacional de los Derechos Humanos (CNDH), 2002, Recomendación General 4/2002 derivada de las prácticas administrativas que constituyen violaciones a los derechos humanos de los miembros de las comunidades indígenas respecto de la obtención de consentimiento libre e informado para la adopción de métodos de planificación familiar.

Comisión Nacional de los Derechos Humanos (CNDH), 2017a, Recomendación General 29/2017 sobre el expediente clínico como parte del derecho a la información en servicios de salud. 
Comisión Nacional de los Derechos Humanos (CNDH), 2017b, Recomendación General 31/2017 sobre violencia obstétrica en el sistema nacional de salud.

Comité de Derechos Económicos, Sociales y Culturales de las NaCiones Unidas (Comité DESC), 2000, Observación General No. 14, sobre "El derecho al disfrute del más alto nivel posible de salud".

Inmujeres, 2010, Población usuaria de servicios de salud, disponible en: http://estadistica.inmujeres.gob.mx/myhpdf/93.pdf.

Moreddu Gilabert, Mariana, 2017, Regulación jurídica del expediente clínico electrónico, México, Tirant lo Blanch.

Roldán Xopa, José, 2014, "Acceso al expediente médico", Derecho a saber: balance y perspectivas cívicas, sección V.

\section{Marco jurídico}

Amparo en Revisión 632/2014, 2014, Unanimidad de votos. Ponente: ministro Alberto Pérez Dayán. Secretaria: Georgina Laso de la Vega Romero.

Caso Albán Cornejo y otros vs. Ecuador, 2007, Corte IDH.

Caso Rodríguez Vera y otros vs. Colombia, 2014, Corte IDH.

INAI, criterio 317/17, 24 de septiembre de 2017.

NOM-004-SSA3-2012, Del Expediente Clínico, México.

Principios de París, Principios relativos al estatuto y funcionamiento de las instituciones nacionales de protección y promoción de los derechos humanos.

Reglamento Interno de la Comisión Nacional de los Derechos Humanos. 